\title{
Relationship between serum bilirubin concentrations and diabetic nephropathy in Shanghai Han's patients with type 1 diabetes mellitus
}

Xu Li ${ }^{\dagger}$, Lei Zhang ${ }^{\dagger}$, Haibing Chen ${ }^{\dagger}$, Kaifeng Guo, Haoyong Yu, Jian Zhou, Ming Li, Qing Li, Lianxi Li, Jun Yin, Fang Liu, Yuqian Bao, Junfeng Han ${ }^{*}$ and Weiping Jia*

\begin{abstract}
Background: Recent studies highlight a negative association between total bilirubin concentrations and albuminuria in patients with type 2 diabetes mellitus. Our study evaluated the relationship between bilirubin concentrations and the prevalence of diabetic nephropathy (DN) in Chinese patients with type 1 diabetes mellitus (T1DM).

Methods: A total of 258 patients with T1DM were recruited and bilirubin concentrations were compared between patients with or without diabetic nephropathy. Multiple stepwise regression analysis was used to examine the relationship between bilirubin concentrations and $24 \mathrm{~h}$ urinary microalbumin. Binary logistic regression analysis was performed to assess independent risk factors for diabetic nephropathy. Participants were divided into four groups according to the quartile of total bilirubin concentrations (Q1, 0.20-0.60; Q2, 0.60-0.80; Q3, 0.80-1.00; Q4, 1.00-1.90 mg/dL) and the chi-square test was used to compare the prevalence of DN in patients with T1DM.

Results: The median bilirubin level was 0.56 (interquartile: $0.43-0.68 \mathrm{mg} / \mathrm{dL}$ ) in the DN group, significantly lower than in the non-DN group (0.70 [interquartile: $0.58-0.89 \mathrm{mg} / \mathrm{dL}$ ], $P<0.001)$. Spearman's correlational analysis showed bilirubin concentrations were inversely correlated with $24 \mathrm{~h}$ urinary microalbumin $(r=-0.13, P<0.05)$ and multiple stepwise regression analysis showed bilirubin concentrations were independently associated with $24 \mathrm{~h}$ urinary microalbumin. In logistic regression analysis, bilirubin concentrations were significantly inversely associated with nephropathy. In addition, in stratified analysis, from the first to the fourth quartile group, increased bilirubin concentrations were associated with decreased prevalence of DN from $21.90 \%$ to $2.00 \%$.
\end{abstract}

Conclusion: High bilirubin concentrations are independently and negatively associated with albuminuria and the prevalence of DN in patients with T1DM.

Keywords: Type 1 diabetes mellitus, Diabetic nephropathy, Bilirubin concentrations

\footnotetext{
* Correspondence: tjhjf@163.com; wpjia@yahoo.com

${ }^{\dagger}$ Equal contributors

Department of Endocrinology and Metabolism, Shanghai Jiao Tong

University Affiliated Sixth People's Hospital, Shanghai Clinical Center for

Diabetes, Shanghai Diabetes Institute, Shanghai Key Laboratory of Diabetes

Mellitus, Shanghai Key Clinical Center for Metabolic Disease, 600Yishan road,

Shanghai 200233, China
} 


\section{Background}

Diabetic nephropathy (DN) is the most common cause of end-stage renal disease worldwide, which remains a major cause of morbidity and mortality in patients with T1DM [1]. Oxidative stress may be a common pathway linking diverse, seemingly distinct, potential mechanisms underlying the pathogenesis of complications in diabetes, including nephropathy [2]. Bilirubin is the end product of haem catabolism and it acts as a powerful biological antioxidant [3, 4]. A study on Gilbert syndrome (GS) reported that the prevalence of ischemic heart disease (IHD) was $2 \%$ in GS patients (who are characterised by high bilirubin concentrations) compared to $12.1 \%$ in the general population, indicating that chronic hyperbilirubinemia prevents the development of IHD by increasing the antioxidant capacity of serum [5]. In a recent study that used the deoxycorticosterone acetate (DOCA)-salt model of hypertension in Heine oxygenase (HO) $-1^{-} /^{-}$and $\mathrm{HO}-1^{+} /^{+}$mice, systolic arterial pressure was significantly elevated in $\mathrm{HO}-1^{-}$mice treated with DOCA salt but not in $\mathrm{HO}-1^{+} /^{+}$mice; in addition, DOCA-salt impaired vasorelaxation was noted in wild-type rats but not in hyperbilirubinemic rats [6]. These results suggest that the $\mathrm{HO}-1$ isozyme and the product bilirubin may have protective effects on vascular disease. This finding has also been confirmed in a model of balloon injury [7]. In addition, diabetic hyperbilirubinemic Gunn $j / j$ rats excrete significantly less urinary albumin than diabetic non-hyperbilirubinemic Gunn j/+ rats and administration of biliverdin $(5 \mathrm{mg} / \mathrm{kg})$ protects against both albuminuria and renal mesangial expansion in $\mathrm{db} / \mathrm{db}$ mice [8]. These findings suggest that bilirubin and biliverdin may protect against DN. A populationbased study showed that high bilirubin concentrations in serum are associated with reduced risk of DN [9]. Furthermore, several longitudinal studies on healthy subjects and type 2 diabetes mellitus (T2DM) also demonstrated that low serum bilirubin concentration could be a novel risk factor for the development of albuminuria in patients with type 2 diabetes [10, 11]. However, renal disease remains a major cause of morbidity and mortality in patients with T1DM and the association between bilirubin concentrations in serum and the prevalence of diabetic nephropathy in patients with T1DM has not yet been studied. Therefore, in the present study, we evaluated the association between bilirubin concentrations in serum and the prevalence of $\mathrm{DN}$, and we hypothesized that bilirubin concentrations may inversely associate with the prevalence of DN in Chinese patients with T1DM.

\section{Methods}

\section{Study population}

This was a cross-sectional, population-based study involving 258 patients with T1DM. Participants were hospitalised patients who presented at the Department of Endocrinology and Metabolism, Shanghai Jiaotong University Affiliated Sixth People's Hospital between January 2008 and January 2013. T1DM was defined as anti-glutamate decarboxylase (GAD) antibody level $\geq 1.5$ $\mathrm{U} / \mathrm{mL}$ and injecting insulin at least three times daily or using an insulin pump, and DN were diagnosed according to KDOQI Clinical Practice Guidelines and Clinical Practice Recommendations for Diabetes and Chronic Kidney Disease for 2007 [12]. Hypertension was diagnosed if the patient had a blood pressure greater than $140 / 90 \mathrm{mmHg}$ or used anti-hypertensive drugs. Subjects were excluded for the following reasons: absence of data (bilirubin, haemoglobin A1c [HbA1c]), abnormal thyroid function (hyperthyroidism or hypothyroidism), elevated serum levels of creatinine $(>124 \mu \mathrm{mol} / \mathrm{L})$, serum bilirubin concentrations $>2.0 \mathrm{mg} / \mathrm{dL}$, and T2DM or specific diabetes. In addition, patients were excluded if they had confounding hepatobiliary or haemolytic disease, hepatitis $\mathrm{B}$ or $\mathrm{C}$, alcoholic liver disease, gallstones, cirrhosis, IgA nephropathy, or urinary tract infections.

\section{Clinical and laboratory measurements}

The clinical parameters investigated included age, height, weight, duration of diabetes, systolic blood pressure (SBP), diastolic blood pressure (DBP), waist circumference (WC), hip circumference, and waist-to-hip ratio (WHR). Biochemical variables were analysed after an overnight fast of at least $10 \mathrm{~h}$ and included fasting plasma glucose (FPG), 2 h postprandial glucose (2hPG), glycated haemoglobin A1c (HbA1c), glycated albumin (GA), C-reactive protein (CRP), 30-min postprandial venous $\mathrm{C}$ peptide, 120-min postprandial venous $\mathrm{C}$ peptide, albumin, aspartate aminotransferase (AST), alamine aminstransferase (ALT), gamma glutamytransferase $(\gamma-G T)$, total cholesterol (TC), triglyceride level (TG), low-density lipoprotein cholesterol (LDL-C), highdensity lipoprotein cholesterol (HDL-C), haemoglobin (Hb), $24 \mathrm{~h}$ urinary microalbumin, total GFR, uric acid, creatinine $(\mathrm{Cr})$, and total bilirubin.

Subject height was measured to the nearest $0.1 \mathrm{~cm}$ with subjects not wearing shoes and weight was measured to the nearest $0.1 \mathrm{~kg}$ while the subjects wore light clothing; BMI was calculated as weight $(\mathrm{kg})$ divided by the square of the height $(\mathrm{m})$. SBP and DBP were measured after participants had rested for at least $5 \mathrm{~min}$. WC (cm) was measured midway between the costal margin and the iliac crest at the end of a normal expiration and the hip circumference was measured as the circumference around both greater femoral trochanters. The WHR was calculated by dividing waist by hip circumference $(\mathrm{cm})$. All biochemical determinations were performed using the same standard laboratory methods. After overnight fasting, blood was drawn early 
in the morning from the antecubital vein into vacuum tubes to determine the concentrations of fasting plasma glucose (FPG), C peptide, CRP, and concentrations of lipid components and liver enzymes. Bilirubin concentrations from serum samples were determined using the vanadate oxidation method.

Measurement of GFR using the ${ }^{99 \mathrm{~m}}$ Tc-diethylene triamine pentaacetic acid $\left({ }^{99 \mathrm{~m}} \mathrm{Tc}-\mathrm{DTPA}\right)$ renal dynamic imaging method, and ${ }^{99 \mathrm{~m}}$ Tc-DTPA renal dynamic imaging (modified Gate's method) was measured by Millennium TMMPR SPECT from General Electric Medical System [13]. Urine albumin excretions were evaluated by calculating total amounts of $24 \mathrm{~h}$ urinary microalbumin. The stage of albuminuria was defined as normal if no more than $30 \mathrm{mg} / 24 \mathrm{~h}$, microalbuminuria if $24 \mathrm{~h}$ urinary microalbumin was $30-299 \mathrm{mg} / 24 \mathrm{~h}$, and macroalbuminuria if $24 \mathrm{~h}$ urinary microalbumin was equal to or higher than $300 \mathrm{mg} / 24 \mathrm{~h} \mathrm{[12]}$.

\section{Statistical analysis}

Data are expressed as the means \pm standard deviation (SD) for a normal distribution of variables or as the median (interquartile range) for a skewed distribution of variables. For continuous data with a normal distribution and a skewed distribution between DN and non-DN patients, unpaired Student's $t$-tests and nonparametric tests, respectively, were used for statistical analyses. Categorical variables were compared using a chi-square test. Spearman's correlational analysis between $24 \mathrm{~h}$ urinary microalbumin, and other variables was performed. Multiple stepwise regression analysis was performed to assess the relationship between bilirubin concentrations and $24 \mathrm{~h}$ urinary microalbumin. Binary logistic regression analysis was performed to assess independent risk factors for diabetic nephropathy. Participants were divided into four groups according to the quartile of total bilirubin concentrations and the chisquare test was used to compare the prevalence of DN in patients with T1DM. The first quartile was $0.20-0.60 \mathrm{mg} / \mathrm{dL}$, second quartile was $0.60-0.80 \mathrm{mg} / \mathrm{dL}$, third quartile was $0.80-1.00 \mathrm{mg} / \mathrm{dL}$, and fourth quartile was $1.00-1.90 \mathrm{mg} / \mathrm{dL}$. All statistical analyses were performed using SPSS version 17.0 for Windows (SPSS, Chicago, IL, USA). A P-value less than 0.05 was considered to indicate statistical significance.

\section{Results}

\section{Patient demographics and laboratory data}

This study included $33 \mathrm{DN}$ and 225 non-DN patients with T1DM. The general and biochemical characteristics of the DN patients (16 males and 17 females) and non-DN patients (111 males and 114 females) are shown in Table 1. The prevalence of patients with diabetic nephropathy, without nephropathy, were $12.8 \%(n=33)$,
$87.2 \%(n=225)$, respectively. Of 33 patients with diabetic nephropathy, 20 were with microalbuminuria and 13 were with macroalbuminuria. The median age was 54 years (34.50-66 years) in DN subjects and 49 years (31-60 years) in non-DN patients. Median bilirubin concentrations were $0.56 \mathrm{mg} / \mathrm{dL}(0.43-0.68 \mathrm{mg} / \mathrm{dL})$ in the DN group and $0.70 \mathrm{mg} / \mathrm{dL}(0.58-0.89 \mathrm{mg} / \mathrm{dL})$ in non-DN patients $(P<0.01)$, a significant difference. $24 \mathrm{~h}$ urinary microalbumin level was significantly higher in the DN group than the non-DN group (196.67 $\mathrm{mg}$ vs. $7.01 \mathrm{mg}, P<0.01)$ and the same trend was observed with the duration of diabetes, SBP, WHR, uric acid, creatinine levels $(P<0.01)$, whereas albumin, haemoglobin, and total GFR levels were lower in the DN group $(P<0.05)$.

\section{Bilirubin concentrations in serum are independently and negatively associated with $\mathbf{2 4} \mathrm{h}$ urinary microalbumin} Previous studies have shown that microalbuminuria can indicate the progression of $\mathrm{DN}$ in patients with T1DM $[14,15]$. To investigate the risk factors associated with microalbuminuria, we performed Spearman's correlation analysis between bilirubin concentrations and other biochemical characteristics. As expected, $24 \mathrm{~h}$ urinary microalbumin was negatively correlated with bilirubin concentrations $(r=-0.13, P<0.05)$, HDL-C $(r=-0.16, P=0.01)$, and total GFR $(r=-0.20, P<0.001)$ and positively correlated with duration of diabetes, BMI, SBP, WHR, CRP, uric acid, Cr, TG levels (all $P<0.001$ ). These results are presented in Table 2. To determine whether bilirubin concentrations were independently correlated with $24 \mathrm{~h}$ urinary microalbumin, we performed multiple stepwise regression analysis. $\mathrm{Cr}(\beta=0.45, P<0.001)$, total bilirubin $(\beta=-0.19, P<0.001)$, SBP $(\beta=0.15, P=0.005)$ levels were independently related to $24 \mathrm{~h}$ urinary microalbumin. This result is shown in Table 3.

\section{Bilirubin concentrations in serum are independently associated with diabetic nephropathy}

The significant $24 \mathrm{~h}$ urinary microalbumin-related findings prompted a binary logistic regression analysis to identify factors that were independently correlated with $\mathrm{DN}$. When $\mathrm{DN}$ was set as the dependent variable and bilirubin concentrations, duration of diabetes, BMI, SBP, DBP, WHR, HbA1c, GA, CRP, Fasting C peptide, FPG, CRP, HDL-C, TG, TC, LDL-C, Albumin, Haemoglobin were set as covariates, bilirubin concentrations were identified as an independent protective factor for $\mathrm{DN}$ $(\mathrm{OR}=0.05,95 \%$ confidence interval $[\mathrm{CI}]: 0.01-0.66)$, while duration of diabetes $(\mathrm{OR}=1.15,95 \% \mathrm{CI}$ : $1.07-1.22)$ and SBP $(\mathrm{OR}=1.05,95 \% \mathrm{CI}: 1.02-1.08)$ were identified as independent risk factors for $\mathrm{DN}$ (all $P<0.01$, Table 4). 
Table 1 Patient demographics and laboratory data

\begin{tabular}{|c|c|c|c|}
\hline & DN $(n=33)$ & Non-DN $(n=225)$ & $P$-value \\
\hline Male: Female & $16: 17$ & 111:114 & 0.98 \\
\hline Age (years) & $54(34.50-66)$ & $49(31-60)$ & 0.06 \\
\hline Duration of diabetes (years) & $13.92(5.50-20)$ & $4.56(1-8)$ & $<0.001^{* *}$ \\
\hline BMI $\left(\mathrm{kg} / \mathrm{m}^{2}\right)$ & 22.67 (21.85-22.75) & $21.85(17.75-33.65)$ & 0.09 \\
\hline SBP (mmHg) & $130(120-140)$ & $120(110-125)$ & $<0.001^{* *}$ \\
\hline $\mathrm{DBP}(\mathrm{mmHg})$ & $79(70-80)$ & $78(70-80)$ & 0.81 \\
\hline WHR & $0.87(0.85-0.94)$ & $0.86(0.83-0.88)$ & $0.01^{*}$ \\
\hline FPG (mmol/L) & $8.24(5.71-11.47)$ & $7.73(6.18-10.70)$ & 0.48 \\
\hline $2 \mathrm{hPG}(\mathrm{mmol} / \mathrm{L})$ & $12.74 \pm 6.73$ & $14.03 \pm 5.57$ & 0.23 \\
\hline $\mathrm{HbA1c}(\mathrm{mmol} / \mathrm{mol})$ & $79(62-95)$ & $79(60-105)$ & 0.68 \\
\hline GA (\%) & $27.50(21.50-31)$ & $27.50(23-34)$ & 0.44 \\
\hline CRP (mg/L) & $1.01(0.66-3.87)$ & $0.68(0.27-2.86)$ & 0.25 \\
\hline Uric acid (mg/dL) & $328(246.50-414.5)$ & $272(223-316.50)$ & $<0.001^{* *}$ \\
\hline $\operatorname{Cr}(\mathrm{mg} / \mathrm{dL})$ & 78 (60-99.50) & $63(52-74)$ & $0.01^{*}$ \\
\hline Fasting C peptide (ng/mL) & $0.19(0.03-1.13)$ & $0.29(0.05-0.80)$ & 0.56 \\
\hline 30-min postprandial venous C peptide $(\mathrm{ng} / \mathrm{mL})$ & $0.1(0.03-0.96)$ & $0.35(0.05-1.17)$ & 0.93 \\
\hline 120-min postprandial venous C peptide $(\mathrm{ng} / \mathrm{mL})$ & $0.08(0.03-1.62)$ & $0.48(0.07-1.58)$ & 0.64 \\
\hline Albumin (g/dL) & $41(37.50-43)$ & $42.44(40-45)$ & $<0.001^{* *}$ \\
\hline $\mathrm{ALT}(\mathrm{U} / \mathrm{L})$ & $16(11-24.5)$ & $17(12-25)$ & 0.56 \\
\hline AST (U/L) & $18(15-24)$ & $19(15-24)$ & 0.42 \\
\hline Y-GT(U/L) & $18(16-23.50)$ & $15(12-22)$ & 0.73 \\
\hline Total Bilirubin (mg/dL) & $0.56(0.43-0.68)$ & $0.70(0.58-0.89)$ & $<0.001^{* *}$ \\
\hline TC (mmol/L) & $4.75 \pm 1.34$ & $4.41 \pm 0.99$ & 0.09 \\
\hline TG (mmol/L) & $1.09(0.87-1.62)$ & $0.88(0.70-1.17)$ & 0.07 \\
\hline $\mathrm{HDL}-\mathrm{C}(\mathrm{mmol} / \mathrm{L})$ & $1.28(0.95-1.66)$ & $1.27(1.10-1.60)$ & 0.59 \\
\hline LDL-C (mmol/L) & $2.69(2.18-3.36)$ & $2.68(2.16-3.39)$ & 0.17 \\
\hline Haemoglobin (g/dL) & $126(109.50-136.5)$ & $130(74-171)$ & $0.04^{*}$ \\
\hline $24 \mathrm{~h}$ urinary microalbumin (mg/24 h) & 196.67(49.27-1123.33) & 7.01 (4.89-10.44) & $<0.001^{* *}$ \\
\hline Total GFR (mL/min) & $81.75(60.35-96.03)$ & 98.95(86.95-107.20) & $<0.001^{* *}$ \\
\hline
\end{tabular}

Data are expressed as the means + standard deviation (SD) for normal distribution variables or as the median (interquartile range) for skewed distribution variables Abbreviations: DN diabetic nephrōpathy, T1DM type 1 diabetes mellitus, BMI body mass index, SBP systolic blood pressure, DBP diastolic blood pressure, WHR waist-to-hip ratio, FPG fasting plasma glucose, $2 h P G 2 \mathrm{~h}$ postprandial glucose, $\mathrm{HbA1c}$ glycated haemoglobin A1c, GA glycated albumin, $C r$ creatinine, $C R P \mathrm{C}$-reactive protein, $A L T$ alanine aminotransferase, AST asparatate aminotransferase, $Y$-GT $Y$-glutamyltranspeptidase, $T C$ total cholesterol, $T G$ triglycerides, $H D L-C$ high-density lipoprotein cholesterol, $L D L-C$ low-density lipoprotein cholesterol, GFR glomerular filtration rate, OR odds ratio, $95 \%$ CI $95 \%$ confidence interval ${ }^{*} P<0.05,{ }^{* *} P<0.01$

\section{Bilirubin concentrations in serum are negatively associated with the prevalence of DN in patients with T1DM}

The prevalence of DN in patients with T1DM for each quartile of bilirubin concentrations is illustrated in Table 5. The first quartile group had the highest prevalence of DN (21.90\%) among the four quartiles. From the second to the fourth quartile group, the prevalence of $\mathrm{DN}$ decreased from $17.10 \%$ to $2.00 \%$ as bilirubin concentrations increased. ORs from the third to fourth quartile were statistically significant compared to the first quartile using logistic regression analyses (all $P<0.05$ ), but not in the second quartile $(P=0.48)$. After adjusting for duration of diabetes, BMI, TG, Albumin, Haemoglobin and WHR, the ORs for the prevalence of DN decreased significantly with the quartiles of bilirubin concentrations ( $P$ for the trend $=0.04$ ). Therefore, we deduced that high bilirubin concentrations in serum (0.80$1.90 \mathrm{mg} / \mathrm{dL}$ ) may be a protective factor for the development of DN in Chinese patients with T1DM.

\section{Discussion}

Several previous studies have reported that high bilirubin concentrations in serum are negatively associated with the 
Table 2 Correlations of 24 h urinary microalbumin with bilirubin concentrations and other characteristics in subjects with T1DM

\begin{tabular}{|c|c|c|}
\hline & \multicolumn{2}{|c|}{$24 \mathrm{~h}$ urinary microalbumin (unadjusted } \\
\hline & $r$ & $P$-value \\
\hline Age & 0.07 & 0.24 \\
\hline Duration of diabetes & 0.18 & $<0.001^{* *}$ \\
\hline BMI & 0.18 & $<0.001^{* *}$ \\
\hline SBP & 0.30 & $<0.001^{* *}$ \\
\hline DBP & 0.08 & 0.23 \\
\hline WHR & 0.30 & $<0.001^{* *}$ \\
\hline FPG & 0.06 & 0.33 \\
\hline $2 \mathrm{hPG}$ & -0.10 & 0.13 \\
\hline $\mathrm{HbA1c}$ & 0.02 & 0.72 \\
\hline GA & -0.05 & 0.45 \\
\hline CRP & 0.19 & $<0.001^{* *}$ \\
\hline Uric acid & 0.23 & $<0.001^{* *}$ \\
\hline $\mathrm{Cr}$ & 0.20 & $<0.001^{* *}$ \\
\hline Fasting C peptide & 0.01 & 0.92 \\
\hline $\begin{array}{l}\text { 30-min postprandial } \\
\text { venous } C \text { peptide }\end{array}$ & -0.02 & 0.80 \\
\hline $\begin{array}{l}120 \text {-min postprandial } \\
\text { venous C peptide }\end{array}$ & -0.02 & 0.74 \\
\hline TC & -0.01 & 0.98 \\
\hline TG & 0.24 & $<0.001^{* *}$ \\
\hline $\mathrm{HDL}-\mathrm{C}$ & -0.16 & $0.01^{*}$ \\
\hline LDL-C & -0.01 & 0.88 \\
\hline Albumin & -0.07 & 0.27 \\
\hline Haemoglobin & -0.03 & 0.65 \\
\hline Total bilirubin & -0.13 & $0.03^{*}$ \\
\hline Total GFR & -0.20 & $<0.001^{* *}$ \\
\hline
\end{tabular}

Abbreviations: T1DM type 1 diabetes mellitus, BMI body mass index, SBP systolic blood pressure, DBP diastolic blood pressure, WHR waist-to-hip ratio, FPG fasting plasma glucose, $2 h P G 2 \mathrm{~h}$ postprandial glucose, $\mathrm{HbA} 1 \mathrm{c}$ glycated haemoglobin A1c, GA glycated albumin, $C r$ creatinine, CRP C-reactive protein $T C$ total cholesterol, TG triglycerides, $H D L-C$ high-density lipoprotein cholesterol, LDL-C low-density lipoprotein cholesterol, GFR glomerular filtration rate "* $P<0.05,{ }^{* *} P<0.01$

Table 3 Multiple stepwise regression analysis showing the variables independently associated with $24 \mathrm{~h}$ urinary microalbumin

\begin{tabular}{lccl}
\hline Independent variable & Standardised $\beta$ & \multicolumn{1}{l}{$\mathrm{t}$} & $P$-value \\
\hline $\mathrm{Cr}$ & 0.45 & 8.34 & $<0.001^{* *}$ \\
Total bilirubin & -0.19 & -3.65 & $<0.001^{* *}$ \\
SBP & 0.15 & 2.85 & $0.005^{* *}$
\end{tabular}

The original model included the duration of diabetes, BMI, SBP, WHR, $\mathrm{Cr}$, uric acid, CRP, TG, total bilirubin, GFR

Abbreviations: $B M I$ body mass index, SBP systolic blood pressure, WHR waist-to-hip ratio, Cr creatinine, CRP C-reactive protein, TG triglycerides, GFR glomerular filtration rate

${ }^{* *} P<0.01$
Table 4 Independent risk factors associated with the diabetic nephropathy in patients with type 1 diabetes

\begin{tabular}{lrrlcl}
\hline & B & Wald & $P$-value & Exp (B) & $95 \% \mathrm{Cl}$ \\
\hline Duration of diabetes (years) & 0.14 & 17.38 & $<0.001^{* *}$ & 1.15 & $1.07-1.22$ \\
SBP (mmHg) & 0.05 & 8.93 & $<0.001^{* *}$ & 1.05 & $1.02-1.08$ \\
Total bilirubin (mg/d) & -3.01 & 5.17 & $0.002^{* *}$ & 0.05 & $0.01-0.66$
\end{tabular}

Variables of the original model included duration of diabetes, BMI, SBP, DBP, WHR, HbA1c, GA, CRP, Fasting C peptide, FPG, CRP, HDL-C, TG, TC, LDL-C, Albumin, Haemoglobin and total bilirubin. Only significant variables are presented Abbreviations: $B M I$ body mass index, SBP systolic blood pressure, WHR waist-to-hip ratio, FPG fasting plasma glucose, HbA1c glycated haemoglobin A1c, GA glycated albumin, CRP C-reactive protein, TC total cholesterol, TG triglycerides, HDL-C highdensity lipoprotein cholesterol, LDL-C low-density lipoprotein cholesterol, CRP Creactive protein, $\mathrm{HDL}-\mathrm{C}$ high-density lipoprotein cholesterol, TG triglycerides, 95\% Cl $95 \%$ confidence interval

${ }^{* *} P<0.01$

incidence of hypertension [16] and T2DM [17]. In addition, a recent study demonstrated that bilirubin concentrations were significantly negatively correlated with $\log$ (UAE) in patients with type 1 diabetes [18]. We performed a cross-sectional study to examine whether bilirubin concentrations in serum are associated with the prevalence of DN in patients with T1DM. We found that bilirubin concentrations were independently and negatively associated with albuminuria and the prevalence of DN in patients with T1DM. We deduced a range of bilirubin concentrations $(0.80-1.90 \mathrm{mg} / \mathrm{dL})$ that may serve as protective factors for the development of $\mathrm{DN}$ in Chinese patients with T1DM and likely represent a pharmacologically attractive target for slowing the development of DN.

We found that bilirubin concentrations and HbA1c level were not relevant $(r=0.06, P=0.334)$ in 258 patients with type 1 diabetes mellitus (33 patients with diabetic nephropathy, 225 patients without diabetic nephropathy). However, a study from Choi SW et al. evaluated the relationship between $\mathrm{HbA1c}$ and bilirubin in 690 patients with type 2 diabetes mellitus and found that bilirubin concentrations were negatively associated with $\mathrm{HbAlc}$, independent of gender, age, and other confounding factors [19]. Because several studies have confirmed that high bilirubin concentrations are inversely associated with insulin resistance [20-22], T1DM is possibly due to $\beta$-cell destruction and leads to absolute insulin deficiency rather than insulin resistance. Furthermore, results from Mianowska B et al. showed that serum total bilirubin concentration is an independent factor inversely associated with HbA1c level in young patients with type 1 diabetes (type 1 diabetes duration of more than 12 months, age from 2 to 18 years) [23], which means that in T1DM, the results are controversial. Thus, we infer that the unsynchronized results of relationship between serum bilirubin concentration and $\mathrm{HbA1c}$ in patients with diabetes mellitus were attributed to the age, duration of diabetes mellitus and glycemic control. Therefore, we admitted that further study should be designed to investigate the 
Table 5 Prevalence of DN in patients with T1DM according to quartiles of serum bilirubin concentrations

\begin{tabular}{lllll}
\hline Bilirubin quartile & Prevalence (\%) & Unadjusted & & Adjusted \\
\cline { 5 - 5 } & & OR (95\% Cl) & P-value & OR (95\% Cl) \\
\hline Q1 $(n=64)$ & 21.90 & 1 (Referent) & & $0.52(0.19-1.41)$ \\
Q2 $(n=76)$ & 17.10 & $0.74(0.32-1.71)$ & 0.48 & $0.24(0.07-0.82)$ \\
Q3 $(n=68)$ & 7.40 & $0.28(0.10-0.84)$ & $0.02^{*}$ & $0.11(0.01-0.92)$ \\
Q4 $(n=50)$ & 2.00 & $0.07(0.01-0.58)$ & $0.01^{*}$ & $0.04^{*}$ \\
P trend for ORs & & & $0.02^{*}$ & $0.02^{*}$ \\
\hline
\end{tabular}

Adjustment based on duration of diabetes, BMI, TG, Albumin, Haemoglobin and WHR

Bilirubin quartiles: Q1, 0.20-0.60 mg/dL; Q2, 0.60-0.80 mg/dL; Q3, 0.80-1.00 mg/dL; Q4, 1.00-1.90 mg/dL

OR odds ratio, $95 \%$ Cl 95\% confidence interval

${ }^{*} P<0.05$

relationship between serum bilirubin concentration and HbA1c in type 1 diabetes mellitus.

Although several studies have been performed, the exact mechanism underlying the negative association between high bilirubin concentrations and the progression of T1DM remains unknown. A previous study demonstrated that diabetic hyperbilirubinemic Gunn $j / j$ rats with high concentrations of unconjugated bilirubin in plasma excreted significantly less urinary albumin than diabetic non-hyperbilirubinemic Gunn $\mathrm{j} /+$ rats, and that administration of biliverdin $(5 \mathrm{mg} / \mathrm{kg})$ protected against both albuminuria and renal mesangial expansion in $\mathrm{db} / \mathrm{db}$ mice. The same authors subsequently discovered that treatment with bilirubin and biliverdin completely inhibited oxidative stress-induced increased expression of NOX4 mRNA and protein levels of cultured vascular endothelial and mesangial cells [8]. These findings suggest that bilirubin and biliverdin significantly inhibit NADPHdependent superoxide production. A study using an isolated, perfused rat kidney model demonstrated that micromolar dose administration of exogenous bilirubin resulted in significantly improved renal vascular resistance, urine output, glomerular filtration rate, tubular function, and mitochondrial integrity after ischemiareperfusion injury (IRI) and showed that bilirubin pretreatment may have future clinical applications, particularly in IRI after organ transplantation [24]. Furthermore, studies on diabetic rats have shown that exogenously administered $\mathrm{CO}$ or bilirubin can protect endothelial cells from oxidative stress-mediated injury [25]. A study on hyperbilirubinemia caused by atazanavir treatment in 16 subjects with T2DM indicated that hyperbilirubinemia is associated with a significant improvement in endothelial function [26]. Considering that oxidative stress, described as increased levels of reactive oxygen species, may be a common pathway linking diverse mechanisms underlying the pathogenesis of complications in diabetes [2] and that hyperglycemia-induced increases in blood pressure and changes in endothelial cells are reversed by antioxidants [27, 28], high concentrations of bilirubin in serum may potentially serve as protective factors against the development of $\mathrm{DN}$ through their antioxidative properties.

This study had several limitations. First, only total bilirubin concentrations in serum were measured, without distinguishing between conjugated versus unconjugated bilirubin. Second, this was a single-center, cross-sectional study, which prevented us from drawing conclusions regarding the temporal nature of the observed association between bilirubin concentrations and the prevalence of $\mathrm{DN}$ in Chinese patients with T1DM. Third, the study population consisted of Chinese males and females and whether our findings apply to other ethnic groups remains unclear.

\section{Conclusion}

In conclusion, our study evaluated the association between total bilirubin and albuminuria, the prevalence of DN in Chinese patients with T1DM. Our data indicate that high bilirubin concentrations in serum may be protective factors for the development of $\mathrm{DN}$.

\section{Abbreviations}

DN: Diabetic nephropathy; GAD: Glutamate decarboxylase; GFR: Glomerular filtration rate; HO: Heine oxygenase; IHD: Ischemic heart disease; T1DM: Type 1 diabetes mellitus

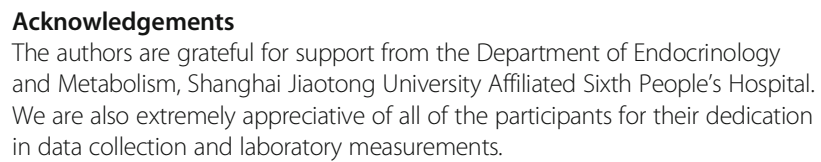

\section{Funding}

This work was supported by a grant the National Natural Sciences Foundation of China (81200564) to Junfeng Han.

\section{Availability of data and materials}

The dataset supporting the conclusions of this article is available from the corresponding author on reasonable request.

\section{Authors' contributions}

JFH and WPJ participated in the design of the study. LZ and HBC performed the statistical analysis. $\mathrm{XL}$ is involved in drafting the manuscript. KFG, HYY, JZ, $M L, Q L, L X L, J Y, F L, Y Q B$ conceived of the study, and participated in its design and coordination and helped to draft the manuscript. All authors read and approved the final manuscript. 


\section{Competing interest}

The authors declare that they have no competing interests.

\section{Consent for publication}

Not applicable.

\section{Ethics approval and consent to participate}

This study was performed according to the principles of the Declaration of Helsinki and was pre-approved by the Ethics Committee of Shanghai Jiaotong University Affiliated Sixth People's Hospital, and all subjects provided written informed consent prior to participating.

\section{Publisher's Note}

Springer Nature remains neutral with regard to jurisdictional claims in published maps and institutional affiliations.

\section{Received: 27 October 2015 Accepted: 24 March 2017}

\section{Published online: 31 March 2017}

\section{References}

1. Raine AE. The rising tide of diabetic nephropathy-the warning before the flood? Nephrol Dial Transplant. 1995;10:460-1.

2. Baynes JW. Role of oxidative stress in development of complications in diabetes. Diabetes. 1991;40:405-12.

3. Stocker R, Yamamoto Y, McDonagh AF, Glazer AN, Ames BN. Bilirubin is an antioxidant of possible physiological importance. Science. 1987:235:1043-6.

4. Balla J, Vercellotti GM, Jeney V, Yachie A, Varga Z, Eaton JW, Balla G. Heme, heme oxygenase and ferritin in vascular endothelial cell injury. Mol Nutr Food Res. 2005:49:1030-43.

5. Vítek L, Jirsa M, Brodanová M, Kalab M, Marecek Z, Danzig V, Novotný L, Kotal P. Gilbert syndrome and ischemic heart disease: a protective effect of elevated bilirubin levels. Atherosclerosis. 2002;160:449-56.

6. Nath KA, d'Uscio LV, Juncos JP, Croatt AJ, Manriquez MC, Pittock ST, Katusic ZS. An analysis of the DOCA-salt model of hypertension in HO-1-/- mice and the Gunn rat. Am J Physiol Heart Circ Physiol. 2007;293:H333-42.

7. Peyton KJ, Shebib AR, Azam MA, Liu XM, Tulis DA, Durante W. Bilirubin inhibits neointima formation and vascular smooth muscle cell proliferation and migration. Front Pharmacol. 2012;3:48.

8. Han SS, Na KY, Chae DW, Kim YS, Kim S, Chin HJ. High Serum Bilirubin Is Associated with the Reduced Risk of Diabetes Mellitus and Diabetic Nephropathy. Tohoku J Exp Med. 2010;221:133-40.

9. Toya K, Babazono T, Hanai K, Uchigata Y. Association of serum bilirubin levels with development and progression of albuminuria, and decline in estimated glomerular filtration rate in patients with type 2 diabetes mellitus. J Diabetes Investig. 2014;5:228-35.

10. Jung CH, Lee MJ, Kang YM, Hwang JY, Jang JE, Leem J, Park JY, Kim HK, Lee WJ. Higher serum bilirubin level as a protective factor for the development of diabetes in healthy Korean men: a 4 year retrospective longitudinal study. Metabolism. 2014;63:87-93.

11. Okada H, Fukui M, Tanaka M, Matsumoto S, Kobayashi K, Iwase H, Tomiyasu K, Nakano K, Hasegawa G, Nakamura N. Low serum bilirubin concentration is a novel risk factor for the development of albuminuria in patients with type 2 diabetes. Metabolism. 2014;63:409-14.

12. KDOQI. KDOQI Clinical Practice Guidelines and Clinical Practice Recommendations for Diabetes and Chronic Kidney Disease. Am J Kidney Dis. 2007:49(2 Suppl 2):S12-154.

13. Gates GF. Split renal function testing using Tc-99 m DTPA. A rapid technique for determining differential glomerular filtration. Clin Nucl Med. 1983:8:400-7.

14. Viberti GC, Hill RD, Jarrett RJ, Argyropoulos A, Mahmud U, Keen H. Microalbuminuria as a predictor of clinical nephropathy in insulindependent diabetes mellitus. Lancet. 1982;1:1430-2.

15. Mogensen CE, Christensen CK. Predicting diabetic nephropathy in insulindependent patients. N Engl J Med. 1984;311:89-93.

16. Chin HJ, Song YR, Kim HS, Park M, Yoon HJ, Na KY, Kim Y, Chae DW, Kim S The bilirubin level is negatively correlated with the incidence of hypertension in normotensive Korean population. J Korean Med Sci. 2009;24(Suppl):S50-6.

17. Ohnaka K, Kono S, Inoguchi T, Yin G, Morita M, Adachi M, Kawate H, Takayanagi R. Inverse associations of serum bilirubin with high sensitivity Creactive protein, glycated hemoglobin, and prevalence of type 2 diabetes in middle-aged and elderly Japanese men and women. Diabetes Res Clin Pract. 2010:88:103-10.

18. Nishimura T, Tanaka M, Sekioka R, Itoh H. Serum bilirubin concentration is associated with eGFR and urinary albumin excretion in patients with type 1 diabetes mellitus. J Diabetes Complications. 2015;29:1223-7.

19. Choi SW, Lee YH, Kweon SS, Song HR, Ahn HR, Rhee JA, Choi JS, Shin MH. Association between Total Bilirubin and Hemoglobin A1c in Korean Type 2 Diabetic Patients. J Korean Med Sci. 2012;27:1196.

20. Song YS, Koo BK, Cho NH, Moon MK. Effect of Low Serum Total Bilirubin levels in serum $(\leq 0.32 \mathrm{mg} / \mathrm{dL})$ on Risk of Coronary Artery Disease in Patients With Metabolic Syndrome. Am J Cardiol. 2014;114:1695-700.

21. Kwak MS, Kim D, Chung GE, Kang SJ, Park MJ, Kim YJ, Yoon JH, Lee HS. Serum bilirubin levels are inversely associated with nonalcoholic fatty liver disease. Clin Mol Hepatol. 2012;18:383.

22. Lin LY, Kuo HK, Hwang JJ, Lai LP, Chiang FT, Tseng CD, Lin JL. Serum bilirubin is inversely associated with insulin resistance and metabolic syndrome among children and adolescents. Atherosclerosis. 2009;203:563-8.

23. Mianowska B, Kamińska A, Fendler W, Szadkowska A, Młynarski W. Bilirubin is an independent factor inversely associated with glycated hemoglobin level in pediatric patients with type 1 diabetes. Pediatr Diabetes. 2014;15:389-93.

24. Adin CA, Croker BP, Agarwal A. Protective effects of exogenous bilirubin on ischemia-reperfusion injury in the isolated, perfused rat kidney. Am J Physiol Renal Physiol. 2005;288:F778-84.

25. Rodella L, Lamon BD, Rezzani R, Sangras B, Goodman Al, Falck JR, Abraham NG. Carbon monoxide and biliverdin prevent endothelial cell sloughing in rats with type I diabetes. Free Radic Biol Med. 2006;40:2198-205.

26. Dekker D, Dorresteijn MJ, Pijnenburg M, Heemskerk S, Rasing-Hoogveld A, Burger DM, Wagener FA, Smits P. The Bilirubin-Increasing Drug Atazanavir Improves Endothelial Function in Patients With Type 2 Diabetes Mellitus. Arterioscler Thromb Vasc Biol. 2011;31:458-63.

27. Marfella R, Verrazzo G, Acampora R, La Marca C, Giunta R, Lucarelli C, Paolisso G, Ceriello A, Giugliano D. Glutathione reverses systemic hemodynamic changes induced by acute hyperglycemia in healthy subjects. Am J Physiol. 1995;268:E1167-73.

28. Curcio F, Ceriello A. Decreased cultured endothelial cell proliferation in high glucose medium is reversed by antioxidants: new insights on the pathophysiological mechanisms of diabetic vascular complications. In Vitro Cell Dev Biol. 1992:28A:787-90.

\section{Submit your next manuscript to BioMed Central and we will help you at every step:}

- We accept pre-submission inquiries

- Our selector tool helps you to find the most relevant journal

- We provide round the clock customer support

- Convenient online submission

- Thorough peer review

- Inclusion in PubMed and all major indexing services

- Maximum visibility for your research

Submit your manuscript at www.biomedcentral.com/submit
Biomed Central 